\title{
Measuring Sustainability: An Application using the Index of Sustainable Functionality in South East Queensland, Australia
}

\author{
Giuseppe T. Cirella, Griffith University, Qld, Australia \\ Longbin Tao, Griffith University, Qld, Australia
}

\begin{abstract}
Over a 25 year span, using five year increments, the index of sustainable functionality (ISF) was applied to measure sustainability within the region of South East Queensland (SEQ), Australia. This study uses functionality by relating it to the level of sustainability within the measured geographical region and utilising methodologies that expand upon the concepts of a dual weightings approach. The dual weightings approach integrates an expert panel and a community-based viewpoint that operate independently of each other to calculate the weightings component of the application. The ISF of $S E Q$ is formulated using novel equations that show higher precision of changes in sustainability via functionality trends. The results of the overall trend for the geographical region of SEQ indicate an intermediate level of functionality which is mostly amounted to related concerns of economic progress and lack of social awareness; while, the natural state of the region is not in severe threat of dysfunctionality which suggests a promising attentiveness to environmental concerns and the like. The use of the ISF in this manner aids in providing a solid basis for achievements and concerns at the community level, offers a historical record as a point of reference as a management tool and presents personal with a view forward on present and future sustainability practices at all levels of government, private enterprise and public institutions. This field of research emphasis' quantitative sustainability, integrated within various levels of qualitative means, can be an important and highlyinfluential piece of the sustainability puzzle.
\end{abstract}

Keywords: Sustainability Science, Sustainable Development, Environmental Engineering, Index of Sustainable Functionality (ISF), Sustainability Indicators

\section{Introduction}

$\mathrm{T}$

THE SCIENCE OF sustainability primarily emerged during the late-1980s from ideas based around concepts of qualitative design; one most notably manuscript during this period is the Bruntland Report (1987). The Bruntland Report was commissioned by the World Commission on Environment and Development and recognised the growing need for a global action plan for change. It pioneered a common definition of sustainable development and put forth the idea that present resource usage must warrant future resource availability. Such an approach was revolutionary in that it globally pioneered the necessity of establishing sustainability goals within an international context (Bruntland, 1987). This first understanding of global sustainability is largely inferred from the interpretation of drastic global events that had and were unfolding at the time. During the late-1980s and early-1990s the way in which the sustainability issue is handled is primarily based on inductive reasoning, that being, the method to investigate the process via qualitative means rather than outcomes or products formulated by quantitative ones. To date, sustainability science continues to endeavour to integrate quantitative sustainability ideas into a new definition of how sustainability can be achieved.
This study utilises a novel approach to defining sustainability via the use of the index of sustainable functionality (ISF). The ISF is an approach founded from inductive reasoning that is principally aimed at using a quantified form of analysis (Cirella and Tao, 2008). This form of analysis has concepts and notions that integrate numerous characteristics from the environmental sustainability index (SamuelJohnston and Esty, 2001), the human development index (Sen, 1985; Sen, 2000; UNDP, 1990; Kelly, 1991), the index for sustainable economic welfare (Daly and Cobb, 1989), the gross happiness indicator (Bhutan RGOP, 1999), the ecological footprint (Rees, 1992) and the genuine progress indicator (GPI) (Cobb et al., 1995). All of these ideas and concepts have spanned the knowledge of sustainability science and have moulded the course for the design of the ISF (Cirella, 2006; Imberger et al., 2007).

The ISF employs an adaptive quantifiable method of measuring sustainability by way of actions via societal performance and rankings. The intension of this study is fundamentally aimed at promoting a cleaner safer society with sustainable higher living standards that support future generations. From this viewpoint it is hopeful that the methodologies utilised in this study can further the knowledge-base of sustainability science and relating fields. 


\section{Project Geography}

The geography of this study is based around the region of South East Queensland (SEQ), Australia. This study utilises the pre- 15 March 2008 amalgamation update of councils within the State of Queensland by dividing SEQ into four regional zones: Northern Sub-Regional Organisation of Councils (NORSROC), Western Sub-Regional Organisation of Councils (WESROC), Southern Regional Organisation of Councils (SouthROC) and Brisbane (Figure 1). There are a total of eighteen councils making up a population of approximately 2.7 million (Queensland Government, 2005). The recent amalgamation update of councils' affects does not change the perimeter boundary of SEQ making the overall results concurrent for future reference.

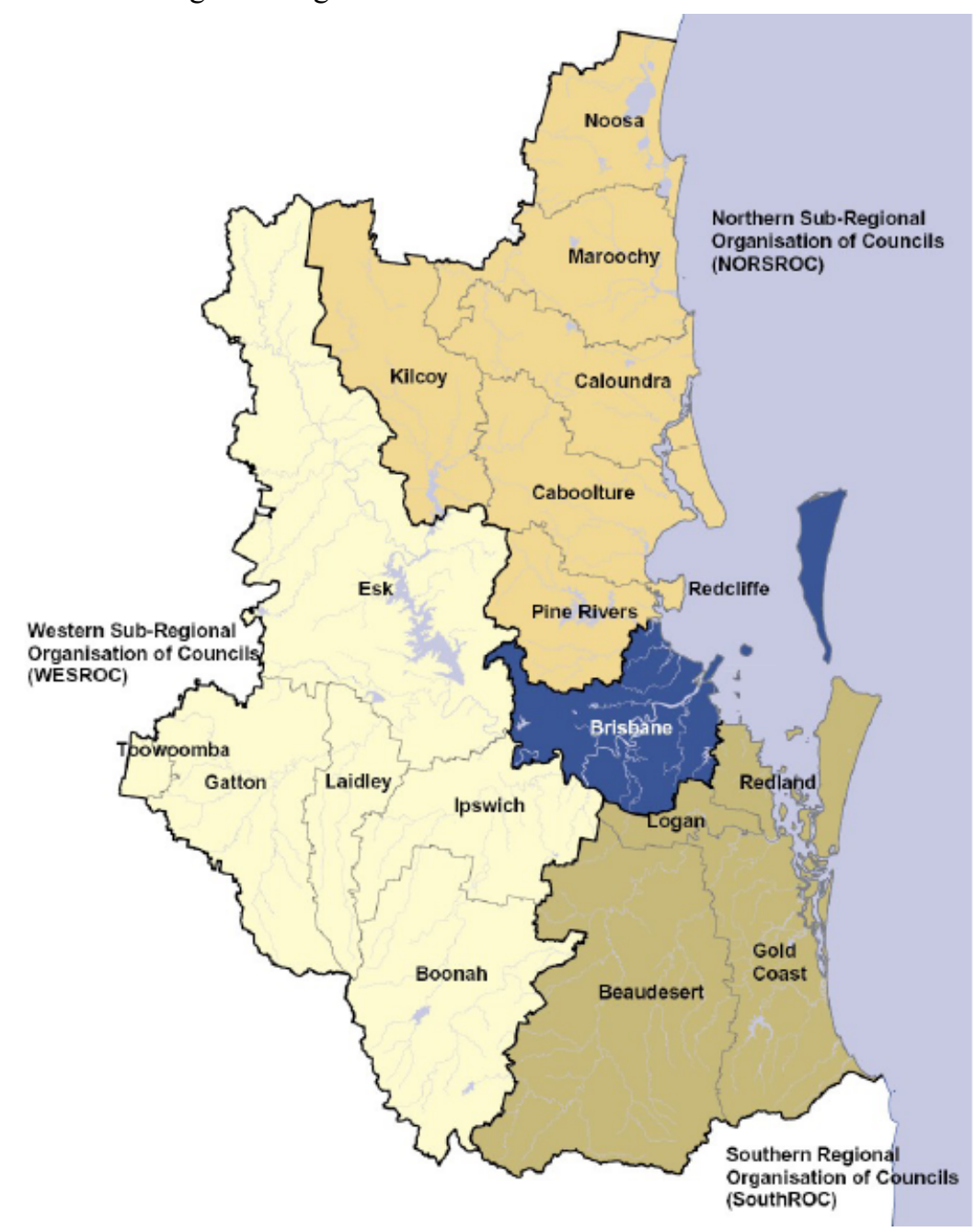

Figure 1: SEQ is Divided in Four Regional Zones and is Made up of Eighteen Councils. This Map Shows the Pre- 15 March 2008 Amalgamation of Council Changes which is used in this Study (Queensland Government, 2005)

This study is labelled the ISF of SEQ; it is one of Australia's fastest growing regions economically, infrastructurally and demographically. SEQ is facing an accelerated influx of people domestically and from outside of Australia - placing a huge burden on its current infrastructure and societal requisites. This need to accommodate such change is accompanying a booming Queensland and Australian economy, making such a scenario an excellent backdrop for the use of an ISF application.

\section{Methodology}

The methodology used for the ISF of SEQ is designed around the premise of an engineering scope and matrix-based technique. It is a quantitative approach of calculating a measure of sustainability via adaptive means of using functional versus dysfunctional indicators that incorporate complex interactions recorded over time to establish a traceable record. The process of contributing and/or being able to adapt to these trends is fundamental to the ability 
to act, or begin to act, sustainability-friendly (Cirella et al., 2007).

The ISF of SEQ is founded on two main parts. The first is the structure of the methodology which is shaped by five steps that identify and define ISF variables. The second part of the methodology is the computation in which the ISF formulae are applied. For reference purposes, to simplify the computational part of the methodology each of the five steps' variables in the methodological structure of the study will be labelled with its associated computational variable(s) beside it in brackets.

\section{Methodological Structure of the Study}

The five steps that identify and define the ISF variables for this study begin with the allocation of the region's domain $(D)$ and the spatial resolution within the domain, referred to as sub-domains $\left(D_{\mathrm{i}}\right)$. The domain of this study is SEQ ( $D$ ); it has four subdomains: NORSROC ( $\left.D_{1}\right)$, WESROC $\left(D_{2}\right)$, SouthROC $\left(D_{3}\right)$ and Brisbane $\left(D_{4}\right)$ (Table 1)

Table 1: The Domain, Sub-Domains, and Systems of the ISF of SEQ

\begin{tabular}{|c|c|c|c|c|}
\hline Domain $(D)$ & $D$ SEQ & & & \\
\hline Sub-domains $\left(D_{\mathrm{i}}\right)$ & $D_{1} \quad$ NORSROC & $D_{2} \quad$ WESROC & $D_{3} \quad$ SouthROC & Brisbane \\
\hline Systems $\left(S_{\mathrm{ij}}\right)$ & $\begin{array}{l}S_{11} \text { NORSROC } \\
\text { Natural } \\
S_{12} \text { NORSROC } \\
\text { Social } \\
S_{13} \text { NORSROC } \\
\text { Individual } \\
S_{14} \text { NORSROC } \\
\text { Economic }\end{array}$ & $\begin{array}{cc}S_{21} \text { WESROC } \\
\text { Natural } \\
S_{22} \text { WESROC } \\
\text { Social } \\
S_{23} \text { WESROC } \\
\text { Individual } \\
S_{24} \text { WESROC } \\
\text { Economic }\end{array}$ & \begin{tabular}{|cc}
$S_{31}$ & SouthROC \\
Natural \\
$S_{32}$ SouthROC \\
Social \\
$S_{33} \quad$ SouthROC \\
Individual \\
$S_{34}$ SouthROC \\
Economic
\end{tabular} & $\begin{array}{c}S_{41} \text { Brisbane } \\
\text { Natural } \\
S_{42} \text { Brisbane } \\
\text { Social } \\
S_{43} \text { Brisbane } \\
\text { Individual } \\
S_{44} \text { Brisbane } \\
\text { Economic }\end{array}$ \\
\hline
\end{tabular}

The second step of the structure of the methodology utilises the matrix-based technique by labelling systems $(S)$ and perspectives $(P)$ on opposite sides of a matrix. The systems of the ISF of SEQ are mechanisms of the domain which jointly relate to all aspects of the region's sustainability. There is the natural system, social system, individual system and economic system. Table 1 shows the numerical values for each of the systems identifying each within their relating project scope. Cross-referencing with the systems are perspectives which are intra or interdomainal viewpoints (Brown, 2006; Cirella, 2006). The perspectives are frequently impacted by the domain in which they are measuring (Imberger et al., 2007) and are the basis for which function selection is made when determining measurement. There are three perspectives: environmental, social and economic. Table 2 shows the system-perspective cross-reference matrix. The perspectives follow the basis of the triple bottom line (TBL) approach that can be positioned relative to one another, "enabling comparison on the basis of substance rather than semantics" (Hacking and Guthrie, 2007). 
Table 2: The System-Perspective Cross-Reference Matrix

\begin{tabular}{|c|c|c|c|c|c|}
\hline \multicolumn{4}{|l|}{ System ( $S$ ) } & & \multirow{5}{*}{ 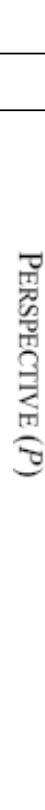 } \\
\hline Natural & Social & Individual & Economic & & \\
\hline $\begin{array}{l}\text { Natural System - } \\
\text { Environmental Per- } \\
\text { spective }\end{array}$ & $\begin{array}{l}\text { Social System - } \\
\text { Environmental Per- } \\
\text { spective }\end{array}$ & $\begin{array}{l}\text { Individual System - } \\
\text { Environmental Per- } \\
\text { spective }\end{array}$ & $\begin{array}{l}\text { Economic System - } \\
\text { Environmental Per- } \\
\text { spective }\end{array}$ & T. & \\
\hline $\begin{array}{l}\text { Natural System - } \\
\text { Social Perspective }\end{array}$ & $\begin{array}{l}\text { Social System-- } \\
\text { Social Perspective }\end{array}$ & $\begin{array}{l}\text { Individual System - } \\
\text { Social Perspective }\end{array}$ & $\begin{array}{l}\text { Economic System - } \\
\text { Social Perspective }\end{array}$ & $\begin{array}{l}0 \\
\varnothing \\
0\end{array}$ & \\
\hline $\begin{array}{l}\text { Natural System - } \\
\text { Economic Perspect- } \\
\text { ive }\end{array}$ & $\begin{array}{l}\text { Social System - } \\
\text { Economic Perspect- } \\
\text { ive }\end{array}$ & $\begin{array}{l}\text { Individual System - } \\
\text { Economic Perspect- } \\
\text { ive }\end{array}$ & $\begin{array}{l}\text { Economic System - } \\
\text { Economic Perspect- } \\
\text { ive }\end{array}$ & 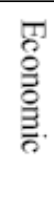 & \\
\hline
\end{tabular}

The third and fourth steps of the methodological structure of the study are related in the sense that the third step identifies functions $(F)$ and relating indicators while the fourth step establishes normalised indicators $(I)$ for the purpose of measurement. "Functions are the activities that a specific system should be carrying out for a particular perspective" (Cirella and Tao, 2008); each relating indicator is thoroughly investigated and data recorded to develop the primary dataset. Relating indicators must associate with their function, must be scientifically valid and be available over time for comparison to thresholds (Cirella et al., 2007; Brown, 2006). In all, the functions totalled 24 while the relating indicators totalled 60. A list of the functions and relating indicators can be found in Cirella and Tao's (2008) research manuscript. All the recorded data is sourced from government reports, interviews, technical papers and relating academic literature. The ISF of SEQ is calculated in five year blocks dating from 1980 to 2005 - making the timeframe of the study 25 years in length. After the relating indicators' data is recorded, normalised indicators are defined using upper and lower functional bounds. The functional bounds are assigned to a value between zero and one to establish a collective standard of measurement between the different scales and units from each relating indicator. "The definition of the upper and lower assigned values equals zero when it is at its worst state and/or situation and one when it is at its best state and/or situation. Thus, when the system operates at full dysfunctionality it is regarded as being at the lowermost level or equal to zero, whereas when the system is at one it is calculated to be at the uppermost level of functionality hence is fully functional" (Cirella et al., 2007).

The fifth step of the structure of the methodology is the implementation of weightings $(W)$ and the aggregation of data. This step is where the structure of the data is put through various statistical weightings before ISF formulation. The weightings of the data are determined using a dual weightings approach. This approach is broken down into two phases, the use of an expert panel and then a community questionnaire. The first phase of the dual weightings approach examines the indicators-to-functions and then the functions-to-perspectives weightings by use of an expert panel. To get these results an expert panel is made up of nine persons from various professional backgrounds all living within the geographical study area. The expert panel members each rank each indicator-to-function and function-to-perspective relationship and decide which of the indicators and functions where most and least related. The relationship between the two reflects the weight assigned that being, the more something is related the more it is significant and thus would bare more weight. The second phase of the dual weightings approach incorporates a community viewpoint by way of a telephone questionnaire. The questionnaire is comprised of twelve questions each representing one question of the system-perspective relationship; it was conducted by the Griffith School of Engineering from 10 April 2007 to 26 May 2007. The twelve questions correspond to the twelve boxes in Table 2 of the cross-reference matrix. For all the councils within SEQ 30 questionnaires were completed, except for Brisbane and the GCCC which totalled 50. 
These two councils had extra questionnaires due to their excessively larger population sizes. The total number of questionnaires conducted within the whole of SEQ totalled 580. The community view is weighted against the perspective-to-system relationship, completing the dual weightings approach.

Aggregation is done by using the percentile weightings on the normalised indicator datasets and then using those values on the weighted normalised indicator datasets. This results in a weighted sum of indicators which allows for the calculation of the normalisation of functions. Once functions have been normalised the percentile weightings of the perspective-to-system is done and the structure of the methodology complete. The aggregation of data is prepared via the weightings from the expert panel and the community viewpoint because it would not be realistic or feasible to conduct a multi-page survey of hundreds of questions to determine indicators' and functions' weightings at the community-level only. Left to an expert panel this task is practical. For the system-perspective relationship the short twelve question questionnaire required approximately a month and a half of telephone calling which allowed a reasonable sample size for the geographical region being studied. In all, this final step is designed to limit the bias and/or subjectivity of the indicators, functions and perspectives.

\section{Computation of the Methodology}

The second part of the methodology is the computational aspect of calculating each structured step to formulate ISF values. The ISF of SEQ is made up of four sub-domains: NORSROC, WESROC, SouthROC and Brisbane. Each of these collaborative groups is made up of councils that each has their own independent ISF value. For the formation of the domain of SEQ eighteen independent ISF values are generated for each council, and divided by eighteen, that is, the total number of councils measured, to create the ISF of SEQ. This regional method is constructed on the basis that each council is equally represented regardless of geographical size, demographic and/or land use. This self-determination approach also backs the structure of the study by focusing on regionalism, an important aspect of sustainability-thinking (Cirella and Tao, 2008). The computation of the ISF of the SouthROC use formulae that first originate from Imberger et al. (2007) but have been significantly modified and expanded in a multitude of ways. The importance of these changes reflects the computational innovation of weightings at the indicator-, function- and perspective-level. This paper also expands upon the Cirella and Tao (2008) formulae by introducing the dual weightings approach making it a significant discovery in utilising the ISF in this manner. The following formulae use the defined variables from Table 3 .

\section{Table 3: Variables of the ISF Model}

$$
\begin{aligned}
& i=\text { sub-domain } \\
& j=\text { system } \\
& k=\text { perspective } \\
& l=\text { function } \\
& m=\text { index or weighting } \\
& r=\text { rank level } \\
& n=\text { total number } \\
& \Lambda=\text { averaged perspective } \\
& \Omega=\text { averaged function } \\
& \Omega=\text { averaged indicator } \\
& B=\text { before value } \\
& A=\text { after value } \\
& \Delta=\text { internal years }
\end{aligned}
$$

The computation of the methodology takes on a sequential method of advancement. Once relating indicators' data is recorded, they must meet the following criteria where the normalisation of data is defined by placing all values equal to and/or between zero and one. This is known as the net normalised indicator value $(I(\lambda k i j l m))$ as defined by Eq. (1). Any value below zero will be equal zero and any value above one will be equal one.

$$
I\left(\lambda_{i j l m}^{k}\right)=\left\{\begin{array}{ccc}
0 & \text { for } & \lambda_{i j l m}^{k}<0 \\
\lambda_{i j l m}^{k} & \text { for } & 0 \leq \lambda_{i j l m}^{k} \leq 1 \\
1 & \text { for } & \lambda_{i j l m}^{k}>1
\end{array}\right.
$$

weightings of certain indicators-to-functions relationships require an approximation when calculating the weighted sum of indicators of mixed datasets; Mixed datasets are datasets that are structured using five year interval and before and after datasets. From these two types of datasets, data that is represented with before and after format use a formula based on linear interpolation (I F Average) Eq. (2). This formula is used strictly to combine these two types of datasets to represent this study's format of using five year interval blocks represented by $\Delta$ from 1980 to 2005. Eq. (2) is executed on the third and seventh function and produces a dataset format calculable for normalisation to its relating function.

$$
I_{F^{\text {Awroge }}}=|A-B| \frac{n \times \Delta}{(A-B,(n=1 \square 4)}
$$

The calculations of the first phase of the dual weightings approach is the formulation of the indicators-to-functions. The weighted functions $(W F)$ Eq. (3) is the first step in this process. This calculates the indicators-to-functions weighted relationship 
from the results based from the expert panel. This is used to define how much weight is given to each indicator representative of its function.

$W_{F}=\frac{\sum_{r=5}^{1} \omega_{r} r}{\max (r)}$

Once the weighted function is ranked, a weighted stack of functions (WF Stack) Eq. (4) is defined. This ranks the number of indicators per function and is the calculation used in the ISF formula. The weighted stack of the function via its relating indicators must equal 100 percent and is calculated by rounding to one decimal place. This concludes the indicators-to-functions formulation.

$$
W_{F^{\text {stack }}}=\operatorname{Round}\left(\frac{W_{F}}{\sum_{r=n}^{1} 100,1}\right)
$$

The formulation of the functions-to-perspectives is similar to the previous two equations except that the variables are at a higher notation. The formulation of the weighted perspective ( $W P$ ) Eq. (5) is similar to the Eq. (3) in that it calculates the functions-toperspective weighted relationship. This formula defines the weight of each function representative of its perspective.

$$
W_{P}=\frac{\sum_{r=5}^{1} \Omega_{r} r}{\sum_{r}}
$$

The weighted stack of the perspectives via its relating functions is the weighted stack of perspectives ( $W$ $P$ Stack) Eq. (6). This is similar to Eq. (4) except it ranks the number of functions per perspective.

$$
W_{P^{\text {Stack }}}=\operatorname{Round}\left(\frac{W_{P}}{\sum_{r=n}^{1} 100,1}\right)
$$

The second phase of the dual weightings approach incorporates the community viewpoint by way of calculating the weightings from the community telephone questionnaire. The weighted system ( $W$
S) Eq. (7) utilises a similar calculation to Eq. (3) and (5) except the notation does not require a weighted stack value. Using the weighted system the results infer, the higher the community response toward an answer the more significant a perspective is to its related system. The weighted system is calculated directly into the ISF formula.

$W_{S}=\frac{\sum_{r=5}^{1} \Lambda_{r} r}{\sum_{r}}$

The summation of each product of the indicators and weightings gives a resulting value by the use of the ISF Eq. (8). The sub-domain value of $i=1$ represents the first of the four sub-domains that are measured as part of the larger project of the ISF of SEQ. The following equation is used on each of the eighteen councils, each giving a resulting ISF value. These values are added together and divided by eighteen to create the ISF values of SEQ.

$\mathrm{ISF}=\sum_{i=1}^{F} \sum_{j=1}^{F_{i}} \sum_{k=1}^{F_{j}} \sum_{l=1}^{F_{i j}^{k}} I\left(\lambda_{i j l m}^{k}\right) W_{F^{\text {Slack }}} W_{P^{\text {Stask }}} W_{S}$

This methodological approach to calculating the ISF of SEQ separates the normalised indicator from the function, the function from the perspective and the perspective from the system. In terms of weightings, it reflects an ability to change as a result of certain priorities. For example, a community may have specific priorities that are different to other communities and/or may have views that change over time. From a methodological standpoint, the ISF integrates such an example, in a quantitative sense with the TBL and to some extent ideas based on capital theory, into an adaptive behavioural manner of assessing recorded data. This study uses environmental, social and economic perspectives which are clearly founded from a TBL framework; to some extent their can also be a link to the systems used as they too share a similar pattern. It is clear the ideas that integrate a TBL framework have assisted in making this study a more robust experiment and well-rounded in a geographical scope. Moreover, upon analysing the linkages among the theories of production, growth, value and distribution it is evident that the concept of capital theory has played a key part in unifying certain qualitative linkages within this study. It should also be noted that the sum of individual perspectives by definition are equal to social ones therefore the individual does not merit a perspective on its own (Cirella and Tao, 2008). To date, this 
methodology innovates on some of the fundamental ISF ideas and offers a new view to defining a measurement of functionality for the purpose of measuring sustainability via a quantitative approach.

\section{Results}

This study spans over a 25 year period and has produced results for each of the eighteen councils within the whole of SEQ. The results of the ISF of SEQ detail an increasing value of 0.444 in 1980 to 0.513 in 2005. This is a functional percentile increase in sustainability within SEQ of $15.54 \%$ over the recorded timeframe. As a comparison to the results found in this study, the ISF has been graphed against the GPI (Cobb et al., 1995) and the gross regional product (GRP); for reference purposes the population growth rate has also been included (Figure 2). This analysis of these three indices shows from 1980 to 1990 that all three follow a similar linear trend of stability; however, over the remainder of the 15 years from 1990 to 2005 there are significant differences in performance. The GPI illustrates a minor increas- ing trend and in many ways parallels the ISF result even though both are calculated in different manners. The ISF is framed in a quantitative method of consequences of expenditure rather than the GPI's calculation of actually expenditure itself (Cirella and Tao, 2008, Cirella et al., 2007; Ranis et al., 2000), making the ISF a more versatile and/or flexible application for analysis and/or for development purposes. From 1990 to 1995 the GRP indicates a percentage increase of $228.88 \%$ in economic expenditure and an even greater percentage increase from 1995 to 2005 of $458.82 \%$. These results indicate the inefficiencies of using an economic-based index like the GRP for measuring the well-being or sustainability of a region due to its failure of over exaggerating economic values. The ISF integrates a cross-reference matrix of points which shows a clearer view of the actual state of functionality in terms its sustainability. As a side note, the population growth rate steadily increasing region-wide at a minor exponential rate which has forced the exponential increase for energy, resources, and land use demands.

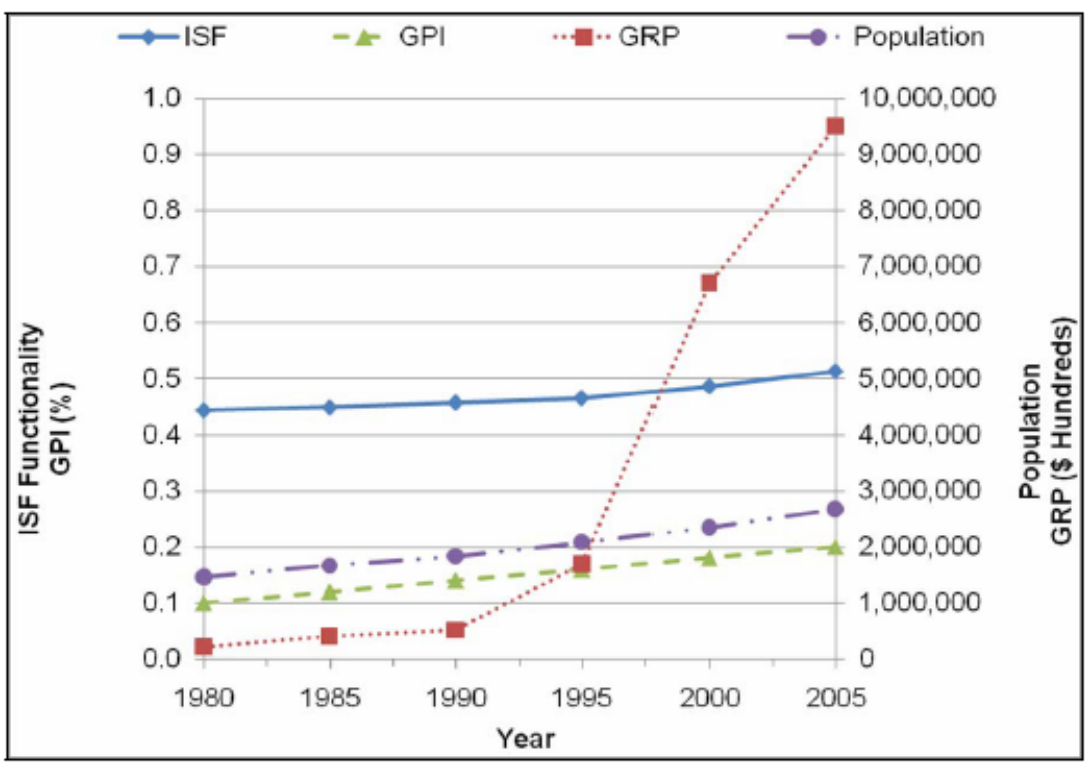

Figure 2: SEQ Graph Showing the Index of Sustainable Functionality (ISF), Genuine Progress Indicator (GPI), Gross Regional Product (GRP) and Population 


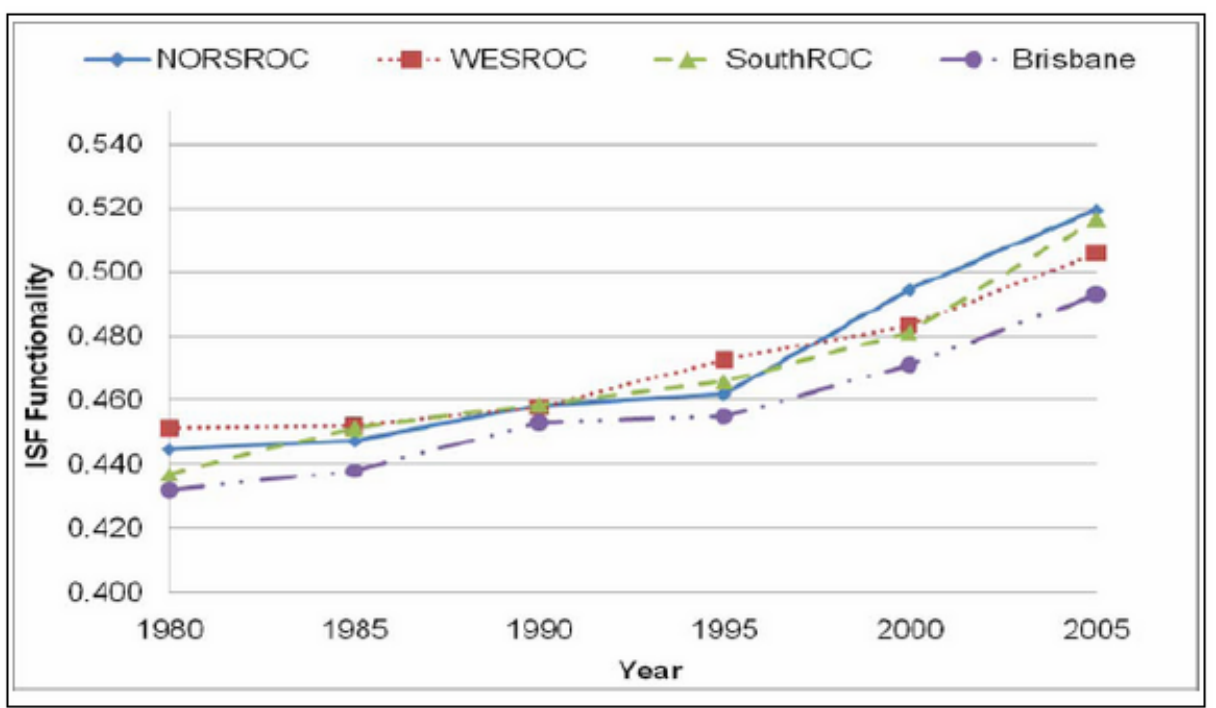

Figure 3: ISF Results for the Four Sub-Domains of NORSROC, WESROC, SouthROC and Brisbane

The four sub-domains show similar ISF results to SEQ. Figure 3 offers a blown up breakdown of the ISF functionality from a sub-domainal viewpoint. These results show an independent increase in each of the four sub-domains of this study. The results all show an incremental increase in each sub-domain for each consecutive five year block recorded. The percentage increases for each sub-domain for the timeframe recorded from 1980 to 2005 are: NORSROC $16.63 \%$, WESROC $12.20 \%$, SouthROC $18.31 \%$ and Brisbane $14.12 \%$. Averaged together is the ISF of SEQ.

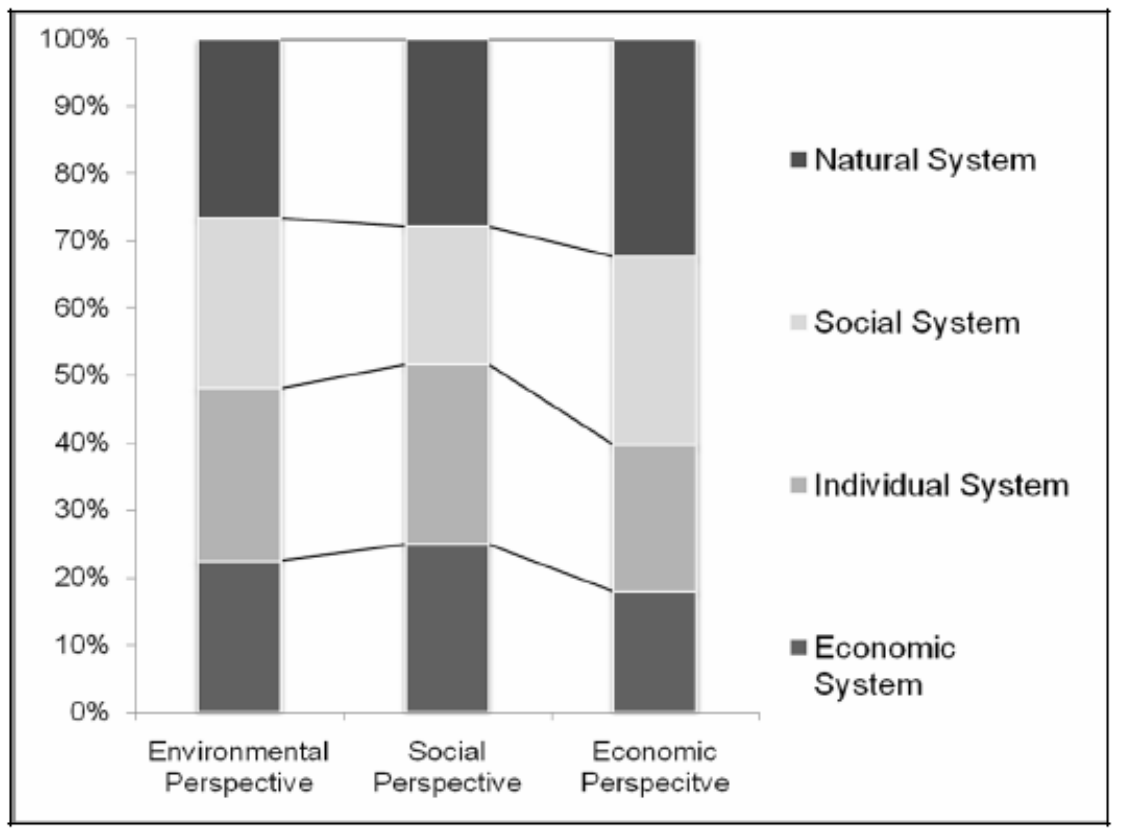

Figure 4: The ISF of SEQ Stacked Percentage of each Perspective Weighted within its Relating System

The results of the weighted functionality of each perspective relative to its system can also be shown. Figure 4 illustrates the three perspectives and the stacked percentage of each perspective weighted across its relating system. This information is relative to the community questionnaire weightings and is similar to Table 2, only that the values shown are project specific and presented in a perspective stacked manner rather than a cross-reference matrix.

\section{Discussion and Concluding Remarks}

From the results of the ISF of SEQ, it is evident that this study has attempted to redefine sustainability in a quantitative manner. In sustainability terms, the results indicate that the functionality of the region is on a slow increase from a quarter century ago. This rise in functionality is evident of the widespread understanding that the issues surrounding sustainability 
need to be addressed from local and state government, business and institutions alike - private and public, volunteer and non-governmental organisations and the community at large.

SEQ shows a significant rise in natural or ecologically-friendly initiatives, which includes numerous natural resource programs region-wide that have an environmental and/or resource-friendly outlook. Almost all of the councils within this study have a large amount of local government resources backing the protection and/or conservation of its locality's environment. This is a positive stance as many other indices do not measure an environment view as thoroughly as the ISF. The social concerns of SEQ are in direct consequence of the booming population that is finding a lack of infrastructure and to some degree issues related to a lack of social awareness. The community has reflected its desire for better community alertness to the sudden changes the region has been and will be facing from this population influx. At an individualistic standpoint the region demonstrates a positive and strong pattern of worth which parallels one of Australia's leading regional economies. SEQ's economic development and economic outlook is expanding with this large demographical change mostly due to investment in local development, tourism and resource care. The economy has also been doing well due to numerous workforce initiatives that accommodate the increasing labour force from its augmenting population. In economic terms, the region does not endure much individualistic difficulties compared to its slight undervalue when dealing with environmentally-economic and/or socio-economic issues. In this case it would be fair to argue that the region would attain an intermediate level of functionality in terms of sustainability.

The ISF approach to looking at recorded data over an extended period of time provides a solid basis for assisting as a management tool. Its ability to measure sustainability in this manner offers a unique approach to a global issue that is on the rise. Through project work similar to this study, the development of a higher standard of living for current and future generations can be prioritised by developing in a unilateral sustainable manner of thinking. This includes providing a solid basis for achievements and concerns at the community level and equal opportunity between different levels of government, private- and public-sectors and within the community itself. The ISF also offers a historical record as a snapshot of the past, as a reference point for the present and as a vision for future sustainability practices. It is hopeful this research can assist in bettering our knowledge-base of what is sustainability and to how such a task might be approached. In all, the continual emergence of sustainability related science is to conform that our current state of development must accord to sustainably-friendly action for present and future generations.

\section{References}

Brown, A., 2006. The index of sustainable functionality (ISF): a prospective tool for assessing the sustainability of the impact of the World Bank projects. Perth, Australia: Centre for Water Research.

Bhutan RGOB., 1999. Gross National Happiness. Thimphu: Royal Government of Bhutan. Retrieved on 11 November 2007 from http://www.bhutanstudies.com/

Bruntland, G. H. (Ed.), 1987. Our Common Future: The World Commission on Environment and Development. Oxford: Oxford University Press.

Cirella, G.T., 2006. The index of sustainable functionality of South East Queensland: an adaptive quantitative method to measure sustainability. RHD Report, Griffith School of Engineering, Griffith University, Gold Coast, Australia.

Cirella, G.T., Tao, L. and Mohamed, S., 2007. An application of an adaptive quantitative method to measure the sustainability of the Gold Coast, Australia. Journal of Coastal Research, SI 50: 52-6.

Cirella, G.T., and Tao, L., 2008. Measuring sustainability: an application of an adaptive quantitative method in Australia. Ecological Economics, (submitted).

Cobb, C., Halstead, T. and Rowe, J., 1995. If the GDP is up, why is America down? Atlantic Monthly, October: 59-78.

Daly, H. and Cobb, J., 1989. For the common good: redirecting the economy toward community, the environment, and a sustainable future. Boston: Beacon Press.

Hacking, T. and Guthrie, P., 2007. A framework for clarifying the meaning of triple bottom-line, integrated, and sustainability assessment. Environmental Impact Assessment, (In press).

Imberger, J., Mamouni, E.A.D., Anderson, J., Ng, M.L., Nicol, S. and Veale, A., 2007. The index of sustainable functionality: a new adaptive, multicriteria measurement of sustainability - application to Western Australia. International Journal of Environment and Sustainable Development, 6(3): 323-55.

Kelly, A.C., 1991. The Human Development Index: handle with care. Population and Development Review, 17(2): 315-24. Queensland Government., 2005. South East Queensland regional plan 2005-2006. Office of Urban Management, Department of Local Government, Planning, Sport and Recreation: The State of Queensland Publication.

Ranis, G., Steward, F. and Ramirez A., 2000. Economic growth and human development. World Development, 28(2): 197219.

Rees, W., 1992. Ecological footprints and appropriated carrying capacity: what urban economics leaves out? Environment and Urbanization, 4(2): 121-30. 
Samuel-Johnston, K. and Esty, D.C., 2001. 2001 Environmental Sustainability Index: an initiative of the global leaders of tomorrow environment taskforce. World Economic Forum. Davos, Switzerland.

Sen, A., 1985. Well-being, agency and freedom: the Dewey Lectures 1984. Journal of Philosophy, 82(4): 169-221.

2000. A decade of human development. Journal of Human Development, 1(1): 17-23.

United Nations Development Programme (UNDP)., 1990. Human Development Report 1990: concept and measurement of human development. Oxford: Oxford University Press.

\section{About the Authors}

Giuseppe T. Cirella

Final year PhD student in engineering. Project work and research includes sustainability, environmental management and social sciences issues.

Dr. Longbin Tao

Griffith University, Australia 